\title{
Estudo Comparativo entre Soluções a 0,5\% de Levobupivacaína, Bupivacaína em Excesso Enantiomérico de $50 \%$ e Bupivacaína Racêmica em Anestesia Peridural para Cirurgia de Abdômen Inferior *
}

\section{Levobupivacaine 0.5\%, 50\% Enantiomeric Excess Bupivacaine and Racemic Bupivacaine in Epidural Anesthesia for Lower Abdominal Procedures. Comparative Study}

Pedro Paulo Tanaka, TSA ${ }^{1}$; Mário Ogleari ${ }^{2}$; Paulo Valmorbida ${ }^{2}$; Maria Aparecida A Tanaka ${ }^{3}$

\section{RESUMO}

Tanaka PP, Ogleari M, Valmorbida P, Tanaka MAA - Estudo Comparativo entre Soluções a 0,5\% de Levobupivacaína, Bupivacaína em Excesso Enantiomérico de 50\% e Bupivacaína Racêmica em Anestesia Peridural para Cirurgia de Abdômen Inferior

JUSTIFICATIVA E OBJETIVOS: Com a finalidade de encontrar um anestésico local mais seguro que a bupivacaína, vários estudos em animais foram realizados com seus isômeros. Este estudo teve como objetivo avaliar a eficácia da bupivacaína em excesso enantiomérico de 50\%, comparada à levobupivacaína e à bupivacaína racêmica, na anestesia peridural em pacientes submetidos à cirurgia de abdômen inferior, pelo período de uma hora após a injeção das soluções.

MÉTODO: Após aprovação pelo Comitê de Ética em Pesquisa, participaram deste estudo, aleatório e duplamente encoberto, 87 pacientes com idade entre 18 e 65 anos, estado físico ASA I e II submetidos à cirurgia de abdômen inferior. Foram distribuídos em três grupos que receberam fracionadamente solução contendo 27 $\mathrm{mL}$ (incluindo a dose-teste) de anestésico local com adrenalina (1:200.000) e fentanil $(100 \mu \mathrm{g})$. O grupo I recebeu solução de levobupivacaína a $0,5 \%$, o grupo II recebeu solução de bupivacaína em excesso enantiomérico de $50 \%$ a $0,5 \%$ e o grupo III recebeu solução de bupivacaína a $0,5 \%$. Os pacientes foram monitorizados por meio de oxímetro de pulso, cardioscópio e pressão arterial não-invasiva. Foram investigadas as características motoras e sensitivas do bloqueio anestésico, bem como a incidência de efeitos colaterais. Os frascos de anestésico local foram preparados sem identificação, numerados e somente ao final do estudo a lista de distribuição aleatória foi aberta.

RESULTADOS: Não foram observadas diferenças significativas com relação à altura e estado físico. Diferença demográfica significativa foi encontrada em relação à idade no grupo $I$. Os parâmetros hemodinâmicos foram semelhantes entre os grupos. Houve diferença significativa em relação à intensidade do bloqueio motor relatado entre os grupos estudados (menor intensidade no grupo I comparada aos grupos II e III).

\footnotetext{
* Recebido do (Received from) Hospital de Clínicas da Universidade Federal do Paraná (UFPr), Curitiba, PR

1. Professor Adjunto da Disciplina de Anestesiologia da UFPr; Co-Responsável do CET do Hospital de Clínicas da UFPr

2. ME 2 do CET do Hospital de Clínicas da UFPr

3. Anestesiologista do Hospital de Clínicas da UFPr

Apresentado (Submitted) em 02 de dezembro de 2004

Aceito (Accepted) para publicação em 09 de agosto de 2005

Endereço para correspondência (Correspondence to)

Dr. Pedro Paulo Tanaka

Rua Justiniano de Mello e Silva, 355

82530-150 Curitiba, PR

E-mail: tanaka@bsi.com.br
}

CONCLUSÕES: Foram observados adequados bloqueios motor e sensitivo para a realização da cirurgia nos grupos estudados com poucos efeitos colaterais, sugerindo que as soluções são eficazes na anestesia peridural para cirurgia de abdômen inferior. A levobupivacaína apresentou menor bloqueio motor que os outros dois grupos.

Unitermos: ANESTÉSICOS, Local: bupivacaína, isômero de bupivacaína, levobupivacaína; TÉCNICAS ANESTÉSICAS, Regional: peridural

\section{SUMMARY}

Tanaka PP, Ogleari M, Valmorbida P, Tanaka MAA - Levobupivacaine $0.5 \%, 50 \%$ Enantiomeric Excess Bupivacaine and Racemic Bupivacaine in Epidural Anesthesia for Lower Abdominal Procedures. Comparative Study

BACKGROUND AND OBJECTIVES: With the purpose of finding a local anesthetic agent safer than racemic bupivacaine, several animal studies have been performed with its isomers. This study aimed at evaluating, for one hour afterinjection, the efficacy of $50 \%$ enantiomeric excess bupivacaine as compared to levobupivacaine and racemic bupivacaine for epidural anesthesia in patients submitted to lower abdominal procedures.

METHODS: After the Research Ethics Committee approval, 87 patients aged 18 to 65 years, physical status ASA I and II, undergoing lower abdominal procedures were included in this randomized double-blind study. Patients were distributed in three groups which received $27 \mathrm{~mL}$ (including test dose) of local anesthetics with epinephrine (1:200,000) and fentanyl $(100 \mu \mathrm{g})$ solutions. Group I was given $0.5 \%$ levobupivacaine, group /I received $50 \%$ enantiomeric excess $0.5 \%$ bupivacaine, and group III received $0.5 \%$ bupivacaine. Monitoring consisted of pulse oximetry, cardioscopy and noninvasive blood pressure. Motor and sensory block profiles were evaluated, in addition to the incidence of side effects. Unidentified local anesthetic vials were numbered, and the randomized distribution list was opened only at the end of the experiment.

RESULTS: There were no significant differences regarding height and physical status between groups. There was a significant age difference in group I. Hemodynamic parameters were similar between groups. Motor block intensity was significantly lower in group I as compared to groups II and III.

CONCLUSIONS: All groups presented adequate motor and sensory block for surgery, with few side effects, suggesting that these solutions are effective for epidural anesthesia for lower abdominal procedures. Levobupivacaine produced less motor block as compared to the other compounds.

Key Words: ANESTHETICS, Local: bupivacaine, bupivacaine isomers, levobupivacaine; ANESTHETIC TECNIQUES, Regional: epidural

(c) Sociedade Brasileira de Anestesiologia, 2005 


\section{INTRODUÇÃO}

$\mathrm{A}^{\mathrm{s}}$ bupivacaína racêmica a $0,5 \%$ tem sido usada com sucesso na anestesia peridural para procedimentos na cavidade abdominal, proporcionando excelente anestesia sensitiva e relaxamento muscular. No entanto, maior ênfase é dada à menor toxicidade sistêmica proporcionada pelo isômero levógiro da bupivacaína ${ }^{1}$, sendo sua eficiência anestésica questionada em alguns relatos em nosso meio ${ }^{2}$. Partindo-se dos isômeros purificados da molécula do anestésico local, pode-se manipular a relação enantiomérica de um composto racêmico. Com este artifício, contribui-se para sua eficácia e diminuição de sua toxicidade potencial, com elevação do índice terapêutico ${ }^{3}$.

Este estudo teve por objetivo a comparação das características da anestesia (bloqueios sensitivo e motor) e repercussões hemodinâmicas com a bupivacaína a $0,5 \%$ em excesso enantiomérico de $50 \%$, a levobupivacaína a $0,5 \%$ e bupivacaína racêmica a $0,5 \%$, no espaço peridural, para procedimentos cirúrgicos no abdômen inferior, durante o período de uma hora após a injeção do anestésico local.

\section{MÉTODO}

Após aprovação do Comitê de Ética em Pesquisa do Hospital de Clínicas da Universidade Federal do Paraná, participaram do estudo, aleatório e duplamente encoberto, 87 pacientes com idade entre 18 e 65 anos, estado físico ASA I e II, submetidas à cirurgia de abdômen inferior. Foram excluídos as pacientes portadores de neuropatia periférica, reação de hipersensibilidade à bupivacaína, septicemia, deformidades da coluna vertebral e hipovolemia não controlada.

As pacientes foram distribuídas em três grupos de acordo com o anestésico local administrado: grupo I- pacientes que receberam levobupivacaína a 0,5\%; grupo II - pacientes que receberam bupivacaína em excesso enantiomérico de $50 \%$ a $0,5 \%$ e grupo III - pacientes que receberam bupivacaína racêmica a $0,5 \%$. Elas receberam a visita pré-operatória na noite anterior à cirurgia, para avaliar suas condições físicas e solicitar a autorização para participarem do estudo. A monitorização constou de eletrocardiografia na derivação $D_{\|}$, medida da pressão arterial não-invasiva e oxímetro de pulso. As soluções de anestésico local utilizadas neste trabalho foram fornecidas pelo Laboratório Cristália, em frascos numerados não-identificados contendo $30 \mathrm{~mL}$. Ao final do estudo foram identificados os grupos.

A punção peridural (espaço $L_{3}-L_{4}$ ) foi realizada com a paciente na posição sentada, utilizando agulha de Tuohy 18G, precedida por infiltração dos planos superficiais com lidocaína a $1 \%$ sem vasoconstritor. O espaço peridural foi identificado por meio da técnica de perda da resistência ao ar na seringa. Após aspiração negativa, $150 \mu \mathrm{g}$ de adrenalina foram adicionados a $30 \mathrm{~mL}$ da solução de anestésico local a ser estudada e $3 \mathrm{~mL}$ desta solução foram utilizados como dose-teste. Logo em seguida, foram adicionados $100 \mu \mathrm{g}$ de fentanil ao restante da solução que foi injetada $(24 \mathrm{~mL})$ de maneira fracionada.
O total de solução no espaço peridural foi de $27 \mathrm{~mL}$ ( $3 \mathrm{~mL}$ da dose-teste somados a $24 \mathrm{~mL}$ da solução). Ao final da injeção da solução foi introduzido um cateter peridural, na sala cirúrgica, permitiu-se a administração de midazolam por via venosa.

Após o término da injeção da solução foram avaliados os seguintes parâmetros: pressão arterial sistólica e diastólica, freqüência cardíaca, nível máximo sensitivo (dermátomo atingido), grau de bloqueio motor pela escala de Bromage ${ }^{4}$, durante os primeiros 30 minutos, antes da cirurgia ser iniciada. Os mesmos parâmetros foram avaliados nos seguintes 30 minutos de cirurgia. Foram anotadas todas as complicações e intercorrências havidas no período peri-operatório. Nas pacientes em que a dose foi insuficiente para realização da cirurgia, foi permitida uma dose adicional de $5 \mathrm{~mL}$ da solução. As complicações, definidas como mediatas, foram abalos musculares, tinitus, sonolência, agitação psicomotora, disritmia cardíaca, convulsão, hipotensão com diminuição de $30 \%$ ou mais da pressão sistólica inicial, freqüência cardíaca menor que 50 bat. $\mathrm{min}^{-1}$. As variáveis selecionadas para análise estatística foram inicialmente submetidas à avaliação de sua distribuição através de testes de normalidade, coeficiente de variação e análise de histogramas. Os testes estatísticos aplicados foram selecionados de acordo com a distribuição das variáveis e seu caráter independente ou dependente, de acordo com as análises realizadas.

Para avaliar as possíveis diferenças de eficiência, de qualidade dos bloqueios sensitivo e motor e de toxicidade ocorridas no período pós-anestésico $(0,10,20,30,45$ e 60 minutos após o bloqueio) entre os três tipos de anestésicos utilizados, foi aplicado o modelo de Análise da Variância (ANOVA) para medidas repetidas, considerando a natureza observacional do estudo e distribuição normal das variáveis. Para realizar a mesma análise somente entre dois tipos de anestésicos foi aplicado o teste paramétrico $t$ de Student para amostras independentes, cumprindo os pressupostos de normalidade e homocedasticidade.

Para avaliar as possíveis diferenças entre os grupos em relação aos testes de sensibilidade e força muscular nos momentos avaliados foi aplicado o teste de Mantel-Haenszel. Para todos foram utilizados os testes bicaudais, considerando que as diferenças poderiam estar distribuídas para ambos os lados da curva, com nível de significância mínimo de 5\%. O tamanho da amostra foi estimado considerando um erro de tipo I de $5 \%$ (alfa) e erro do tipo II de 10\%, com um poder de teste estimado mínimo de $90 \%$.

\section{RESULTADOS}

Os pacientes incluídos no estudo foram distribuídos aleatoriamente em 3 grupos: grupo I - bloqueio realizado com levobupivacaína ( $n=35)$; grupo II - bloqueio realizado com bupivacaína em excesso enantiomérico de $50 \%(n=33)$ e grupo III - bloqueio realizado com bupivacaína racêmica $(n=19)$. $\mathrm{Na}$ tabela I estão apresentadas as características desta população de estudo. 
Foi observada diferença estatística significativa entre os grupos estudados, considerando estas variáveis somente em relação à idade $(p=0,005)$.

Tabela I - Características da População

\begin{tabular}{lcccc}
\hline & $\begin{array}{c}\text { Grupo I } \\
(\mathrm{n}=35)\end{array}$ & $\begin{array}{c}\text { Grupo II } \\
(\mathrm{n}=33)\end{array}$ & $\begin{array}{c}\text { Grupo III } \\
(\mathrm{n}=19)\end{array}$ & $\mathrm{p}$ \\
\hline Idade (anos) & $45,11+9,47$ & $39,12+8,24$ & $38,68+6,49$ & $0,005^{*}$ \\
Peso (kg) & $68,0,8+8,66$ & $69,87+15,29$ & $69,31+11,34$ & $0,980^{*}$ \\
Altura (m) & $161,57+4,39$ & $159,81+5,99$ & $159,89+4,82$ & $0,313^{*}$ \\
\hline
\end{tabular}

*ANOVA

Em relação ao estado físico foi observado que no grupo I $22,86 \%$ dos pacientes foram classificados como ASA I e $77,14 \%$, como ASA II. Estes valores foram de 48,48\% e $51,52 \%$, respectivamente para o grupo II e $57,89 \%$ e 42,11 , para o grupo III. No grupo I observou-se maior freqüência de estado físico ASA II, diferindo dos grupos II e III onde a distribuição entre os estados físicos I e II foi mais equivalente $(p=0,02)$.

Os procedimentos cirúrgicos mais freqüentemente realizados foram histerectomia abdominal $(75,59 \%)$ e laqueadura $(14,92 \%)$. A doença associada mais registrada foi hipertensão arterial sistêmica em $31,03 \%$ dos casos.

Na avaliação da pressão arterial sistólica foi observado que nos três grupos estudados houve diminuição dos níveis pressóricos no período pós-anestésico, especialmente nos primeiros 20 minutos, existindo diferença estatística significativa entre os grupos em relação à medida inicial $(p=0,006)$ (Figura 1).

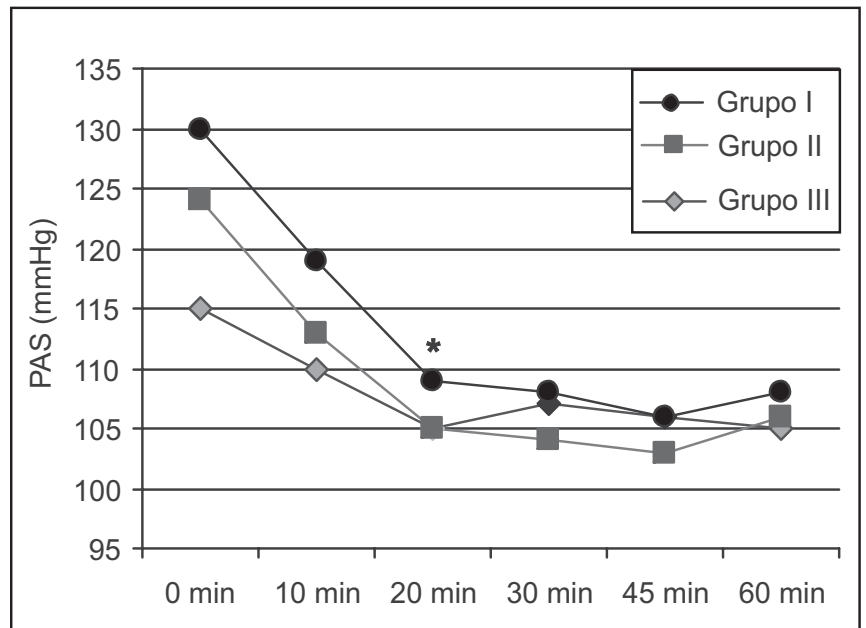

Figura 1 - Variação da Pressão Arterial Sistólica entre os Grupos Estudados

* ANOVA

Em relação à pressão arterial diastólica foi observada, da mesma forma, diminuição dos níveis pressóricos nos primeiros 20 minutos, sem diferença estatística significativa entre os grupos $(p=0,15)$ (Figura 2$)$ em relação à medida inicial.

Revista Brasileira de Anestesiologia

Vol. 55, № 6, Novembro - Dezembro, 2005

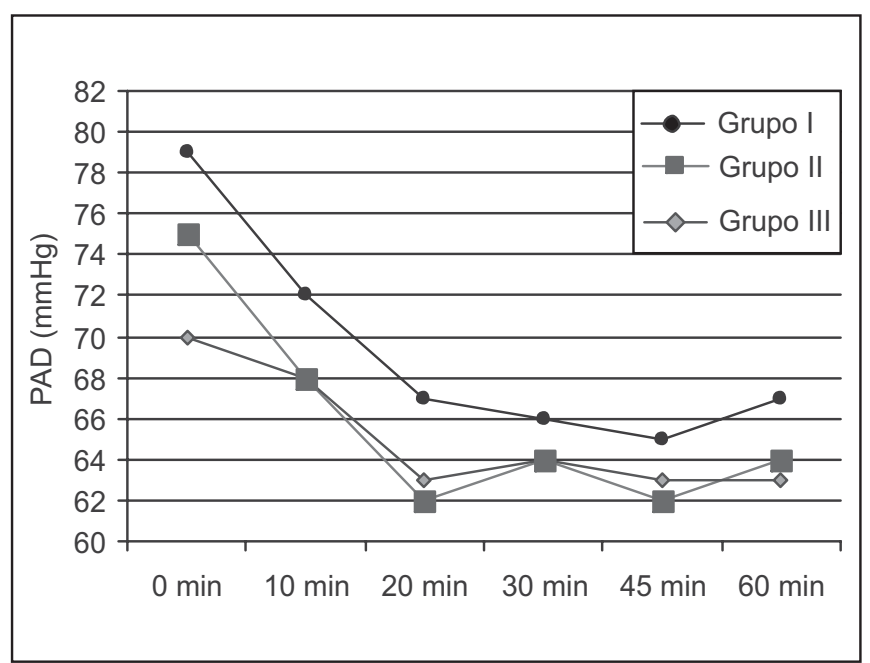

Figura 2 - Variação da Pressão Arterial Diastólica nos Grupos Estudados

Na análise da variação da freqüência cardíaca (Figura 3) foi observado que, embora o grupo II tenha apresentado uma elevação maior aos 10 minutos, não existiu diferença estatística significativa entre os grupos em todo o período pós-anestésico avaliado $(p=0,619)$.

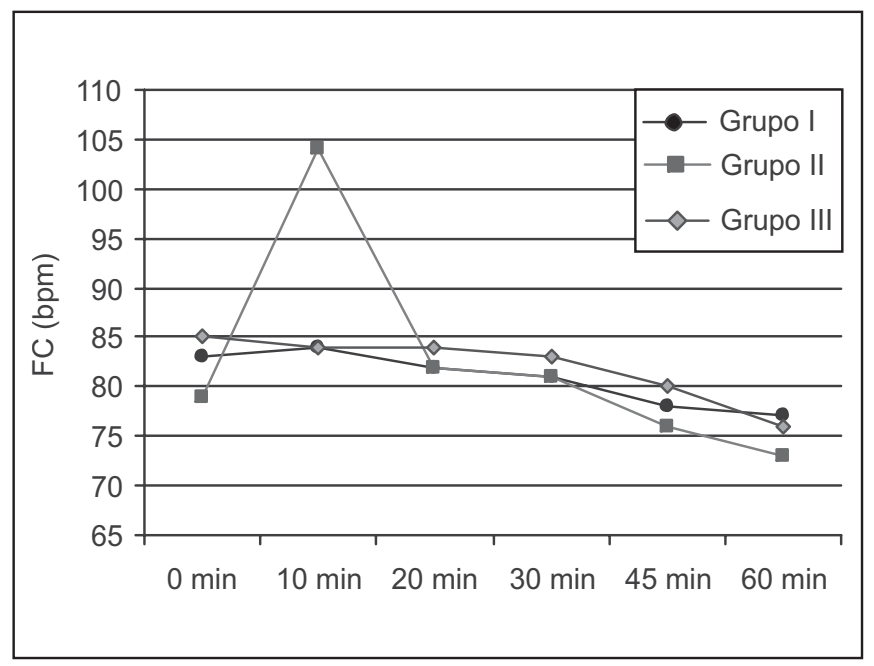

Figura 3 - Variação da Freqüência Cardíaca nos Grupos Estudados

O relaxamento muscular foi avaliado pelo método de Bromage (Figura 4) aos 30 minutos após injeção do anestésico local. Houve diferença estatística significativa do bloqueio grau 2 de Bromage entre os grupos II e III em relação ao grupo I. 


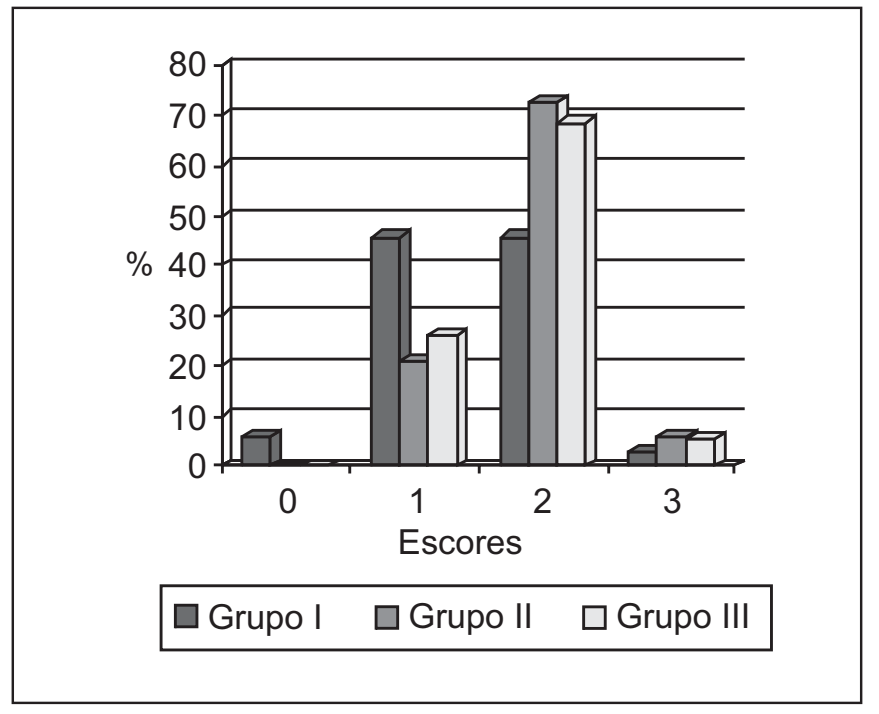

Figura 4 - Graus de Bloqueio Motor (Bromage) aos 30 Minutos

$\mathrm{Na}$ avaliação da extensão do bloqueio sensitivo não houve diferença significativa entre os grupos ao longo do tempo (Figura 5).

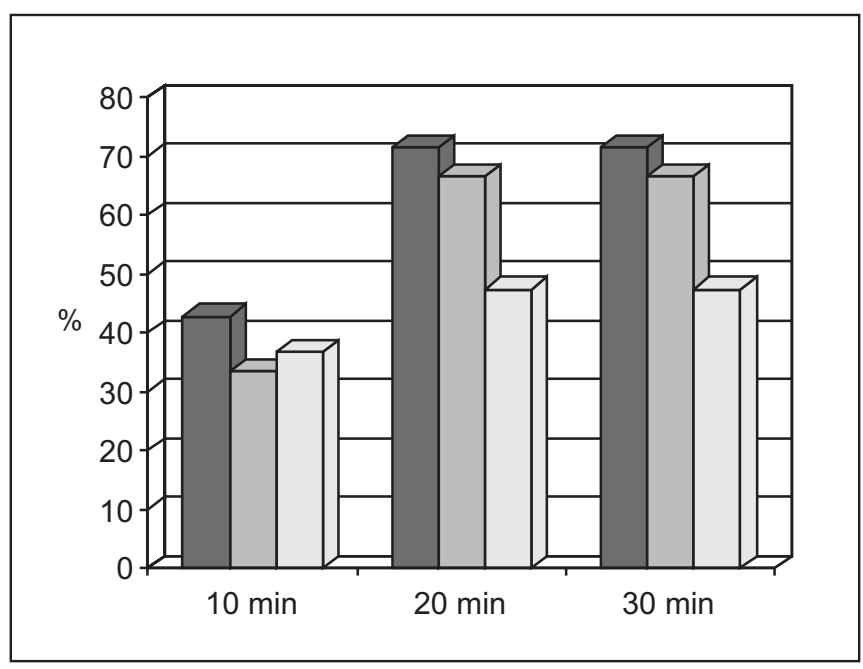

Figura 5 - Avaliação do Bloqueio Sensitivo acima de $T_{4}$

Cinco pacientes no grupo I, três pacientes no grupo Il e duas pacientes no grupo III receberam efedrina por apresentarem diminuição da pressão arterial em níveis pré-definidos para tratamento. O número de falhas para realização da cirurgia foi de três pacientes no grupo I, uma paciente no grupo II e duas pacientes no grupo III. As falhas foram parciais, havendo complementação com dose adicional e sedação em todas as pacientes.

\section{DISCUSSÃO}

Neste estudo foi observada a homogeneidade demográfica entre os grupos com exceção da idade sendo esta maior no grupo I. Embora este dado seja provavelmente casual em razão do tamanho da amostra, a idade, como fator isolado, apresenta pouca influência no bloqueio peridural em adultos, mas ganha importância, quando se considera a população de crianças e idosos ${ }^{5}$.

Na avaliação dos parâmetros hemodinâmicos não houve diferença estatística significativa após 10 minutos da aplicação do anestésico local. A diferença encontrada no valor inicial da pressão arterial sistólica entre os grupos deveu-se provavelmente ao fato de as pacientes não terem recebido medicação pré-anestésica. A pressão arterial sistólica, diastólica, e a freqüência cardíaca demonstraram diminuição semelhante, como era de se esperar, pelo extenso bloqueio da cadeia simpática, conforme demonstrado pelo nível do bloqueio sensitivo observado. Algumas pacientes em cada grupo necessitaram do uso de efedrina para tratar a hipotensão arterial. Os resultados são semelhantes a outros estudos que empregaram a solução com excesso enantiomérico de $50 \%$ de bupivacaína em anestesia peridural lombar ${ }^{6}$ ou torácica ${ }^{7}$.

A adição de adrenalina à solução de anestésico local promove vasoconstrição local interferindo na velocidade de absorção do fármaco injetado, fazendo com que este permaneça mais tempo em contato com as raízes nervosas ${ }^{5}$. Entretanto, estudo realizado por Newton e col. demonstrou maior atividade vasoconstritora com isômero levógiro, fator considerado responsável pela longa duração do bloqueio sensitivo ${ }^{8}$. No presente estudo estabeleceu-se que as soluções seriam administradas com a adição de vasoconstritor em todos os grupos em função dos pesquisadores não terem conhecimento do anestésico local que estava sendo utilizado.

De acordo com estudos envolvendo o isômero levógiro e a bupivacaína em excesso enantiomérico de $50 \%$ em pacientes submetidas à cesariana ${ }^{9-11}$, a associação de opióide ao anestésico local, potencializa seu efeito permitindo a realização de cirurgias envolvendo a cavidade abdominal. Outro recurso descrito ${ }^{12}$ é o aumento da concentração do anestésico local para obtenção do mesmo efeito, embora isto possa acarretar a exposição do paciente a uma maior massa anestésica, diminuindo as vantagens do uso de anestésico local de menor toxicidade sistêmica. No presente trabalho o uso de fentanil e mesmo a introdução do cateter peridural, para a injeção de dose adicional de anestésico local, não evitaram que houvesse falhas de bloqueio, que podem estar relacionadas ao fato de a anestesia ter sido executada por médicos em especialização.

Comparativamente à solução racêmica de bupivacaína, estudo ${ }^{13}$ in vitro demonstrou que o isômero dextrógiro apresenta maior potência $(1,36)$; o isômero levógiro apresenta menor potência $(0,45)$ e que a solução com excesso enantiomérico de $50 \%$ de bupivacaína apresenta potência semelhante $(0,88)$. Entretanto, o uso clínico dos anestésicos locais é Vol. 55, N 6, Novembro - Dezembro, 2005 
mais complexo do que o demonstrado em animais, sendo que as potências in vivo não se correlacionam às encontradas in vitro ${ }^{14}$. Os fatores locais que afetam a difusão e a extensão do anestésico local representam fator de grande impacto sobre os efeitos clínicos. O uso clínico pode não exigir supressão absoluta do potencial de ação, mas somente a interrupção da informação codificada pelo padrão de despolarização, sendo a potência relativa da bupivacaína racêmica e da levobupivacaína no espaço peridural a mesma ${ }^{15}$. Estes dados validam a correlação clínica apresentada neste estudo, onde os anestésicos locais são comparados clinicamente no espaço peridural em concentrações e volumes iguais. Neste estudo foi estabelecido que a cirurgia somente seria iniciada após 30 minutos para que fosse possível a determinação do grau de relaxamento muscular. O tempo médio de 20 minutos foi necessário para atingir o grau máximo de bloqueio motor quando comparado às soluções de bupivacaína racêmica e bupivacaína em excesso enantiomérico de $50 \%$ ${ }^{16}$. Em estudo realizado por $\mathrm{Cox}^{17}$ os resultados foram semeIhantes entre as soluções de levobupivacaína e bupivacaína racêmica a $0,5 \%$. A diferença encontrada em relação à intensidade de bloqueio motor foi significativa, sendo maior o percentual de pacientes que apresentaram Bromage 2 nos grupos II e III, comparados ao grupo I. Delfino e col. ${ }^{18}$, estudando o isômero levógiro puro a $0,5 \%$, comparado com a mistura com excesso enantiomérico de $50 \%$ em pacientes submetidos à cirurgia de varizes, também observaram menor grau de relaxamento, no entanto a diferença foi significativa em relação aos graus 0 e 3 de Bromage. Este fato pode ser justificado pela maior casuística empregada no presente estudo.

Em conclusão, a levobupivacaína bem como a bupivacaína em excesso enantiomérico de $50 \%$ constituem soluções de anestésico local compatíveis para realização de cirurgias de abdômen inferior, comparáveis clinicamente à bupivacaína racêmica. A levobupivacaína apresentou menor bloqueio motor comparado às duas outras soluções.

\section{Levobupivacaine $0.5 \%$, 50\% Enantiomeric Excess Bupivacaine and Racemic Bupivacaine in Epidural Anesthesia for Lower Abdominal Procedures. Comparative Study}

Pedro Paulo Tanaka, TSA, M.D.; Mário Ogleari, M.D.; Paulo Valmorbida, M.D.; Maria Aparecida A Tanaka, M.D.

\section{INTRODUCTION}

Racemic $0.5 \%$ bupivacaine has been successfully used in epidural anesthesia for abdominal procedures, producing excellent sensory anesthesia and muscle relaxation. However, greater emphasis have been given to lower systemic toxicity promoted by levogyrous bupivacaine ${ }^{1}$, though its anesthetic effectiveness has been questioned in some reports ${ }^{2}$.

Starting from purified local anesthetic molecule isomers, it is possible to manipulate the enantiomeric ratio of a racemic compound. This might contribute to its effectiveness and lower potential toxicity, with increased therapeutic index ${ }^{3}$. The purpose of this study was to compare the anesthetic characteristics (sensory and motor block) and hemodynamic repercussions of $50 \%$ enantiomeric excess $0.5 \%$ bupivacaine, $0.5 \%$ levobupivacaine and $0.5 \%$ racemic bupivacaine in the epidural space for lower abdominal procedures, during one hour after local anesthetic injection.

\section{METHODS}

After the Research Ethics Committee, Hospital de Clinicas, Universidade Federal do Paraná approval, 87 patients aged 18 to 65 years, physical status ASA I and II, undergoing lower abdominal procedures, were included in this randomized double-blind study. Exclusion criteria were peripheral neuropathy, hypersensitivity to bupivacaine, sepsis, spinal deformities and uncontrolled hypovolemia.

Patients were distributed into three groups according to the administered drug: group I - $0.5 \%$ levobupivacaine; group II $50 \%$ enantiomeric excess $0.5 \%$ bupivacaine; group III - $0.5 \%$ racemic bupivacaine. Preoperative evaluation was performed the night before surgery when physical conditions were assessed and permission to participate in the study was requested. Monitoring consisted of ECG at $D_{\|}$lead, noninvasive blood pressure and pulse oximetry. Local anesthetic solutions used in this study were supplied by Laboratório Cristália in $30 \mathrm{ml}$ unidentified and numbered vials. Groups were identified at the end of the study.

Epidural puncture ( $\mathrm{L}_{3}-\mathrm{L}_{4}$ interspace) was performed with patients in the sitting position with a 18G Tuohy needle, preceded by superficial tissues infiltration with $1 \%$ plain lidocaine. Epidural space was identified by the loss of resistance to air technique. After negative aspiration, $150 \mu \mathrm{g}$ epinephrine were added to $30 \mathrm{ml}$ of the local anesthetic solution to be injected, and $3 \mathrm{ml}$ of this solution were used as test dose. After that, $100 \mu \mathrm{g}$ fentanyl were added to the remaining solution, $24 \mathrm{ml}$ of which were injected fractionally. The total amount administered was $27 \mathrm{ml}$ ( $3 \mathrm{ml}$ test dose plus $24 \mathrm{ml} \mathrm{so-}$ lution). An epidural catheter was inserted after the solution injection and intravenous midazolam was allowed during the surgery.

After the epidural injection, the following parameters were evaluated: systolic and diastolic blood pressure, heart rate, upper sensory block level (involved dermatomes), and motor block degree by Bromage scale ${ }^{4}$ for 30 minutes before surgery. The same parameters were evaluated during the next 30 surgical minutes.

All perioperative complications and intercurrences were recorded. Additional $5 \mathrm{ml}$ solution dose was allowed for patients in whom the initial dose was insufficient for the procedure. Mediate complications were defined as muscle movements, 
tinnitus, sleepiness, psychomotor agitation, arrhythmia, seizure, hypotension (30\% or more decrease in baseline systolic pressure) and heart rate fall below 50 beat. $\mathrm{min}^{-1}$. Data to be statistically analyzed were initially submitted to distribution evaluation through normality tests, variation coefficient and histogram analysis. Statistical tests were selected according to variables distribution and their dependent or independent character, according to the previously performed analyses.

To evaluate possible differences in anesthetic efficiency, sensory and motor block quality and toxicity $(0,10,20,30$, 45 and 60 minutes after blockade) between the three anesthetics used, Analysis of Variance (ANOVA) for repetitive measures was applied, considering the observational nature of the study and the normal distribution of variables. To perform the same analysis between 2 anesthetics, Student's $t$ parametric test for independent samples was applied, complying with normality and homocedasticity assumptions.

Mantel-Haenszel test was used to evaluate possible differences between groups in sensitivity and muscle strength during evaluated moments.

Two-tailed approaches were used for all tests, considering that differences could be distributed to both sides of the curve, with significance level established to $5 \%$.

Sample size was calculated considering 5\% type I error (alpha) and $10 \%$ type II error, with minimum estimated test power of $90 \%$.

\section{RESULTS}

Patients were randomly distributed in three groups: group I blockade with $0.5 \%$ levobupivacaine $(n=35)$; group II - blockade with $50 \%$ enantiomeric excess $0.5 \%$ bupivacaine $(n=$ 33 ); group III - blockade with $0.5 \%$ racemic bupivacaine $(n=$ 19). Table I shows demographic data of studied population.

Table I - Demographic Data

\begin{tabular}{lcccc}
\hline & $\begin{array}{c}\text { Group I } \\
(\mathrm{n}=35)\end{array}$ & $\begin{array}{c}\text { Group II } \\
\mathrm{n}=33)\end{array}$ & $\begin{array}{c}\text { Group III } \\
(\mathrm{n}=19)\end{array}$ & $\mathrm{p}$ \\
\hline Age (years) & $45.11 \pm 9.47$ & $39.12 \pm 8.24$ & $38.68 \pm 6.49$ & $0.005^{*}$ \\
Weight $(\mathrm{kg})$ & $68.08 \pm 8.66$ & $69.87 \pm 15.29$ & $69.31 \pm 11.34$ & $0.980^{*}$ \\
Height $(\mathrm{m})$ & $161.57 \pm 4.39$ & $159.81 \pm 5.99$ & $159.89 \pm 4.82$ & $0.313^{*}$ \\
\hline
\end{tabular}

*ANOVA

It was observed a statistically significant age difference between groups $(p=0.005)$.

Regarding physical status, $22.86 \%$ of group I patients were classified as ASA I and $77.14 \%$ as ASA II. These values were $48.48 \%$ and $51.52 \%$ for group II and $57.89 \%$ and $42.11 \%$ for group III, respectively. Group I had higher incidence of physical status ASAII, different from groups II and III where distribution of physical status I and II was more equivalent $(p=0.02)$.

Most frequent procedures were abdominal hysterectomy $(75.59 \%)$ and tubal ligature (14.92\%). Most frequent associated disease was systemic hypertension, present in $31.03 \%$ of patients.

There was a post-anesthesia decrease in systolic blood pressure for all groups, especially during the first 20 minutes, with statistically significant difference among groups as compared to baseline values $(p=0.006)$ (Figure 1).

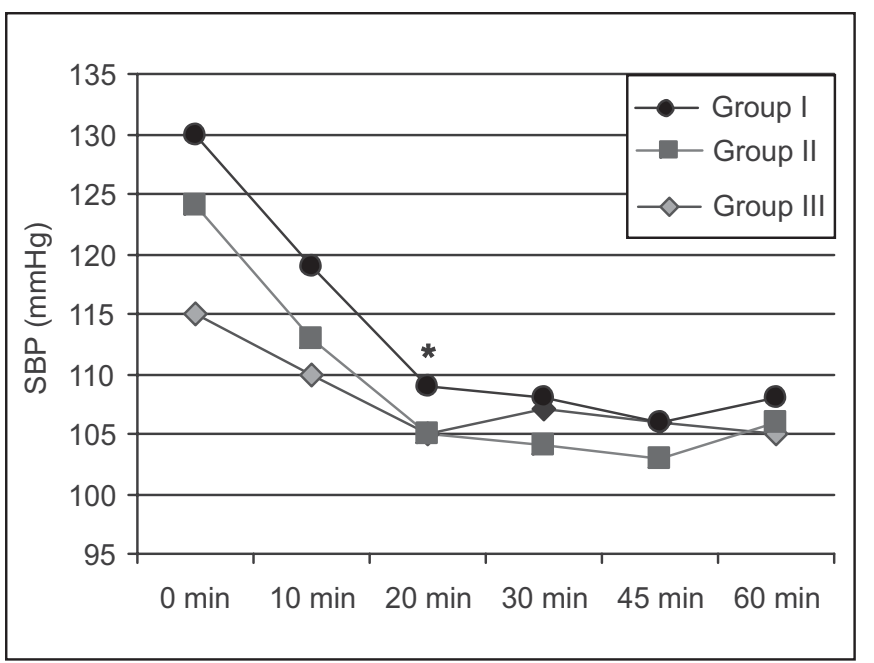

Figure 1 - Systolic Blood Pressure Variation among Studied Groups ${ }^{*}$ ANOVA

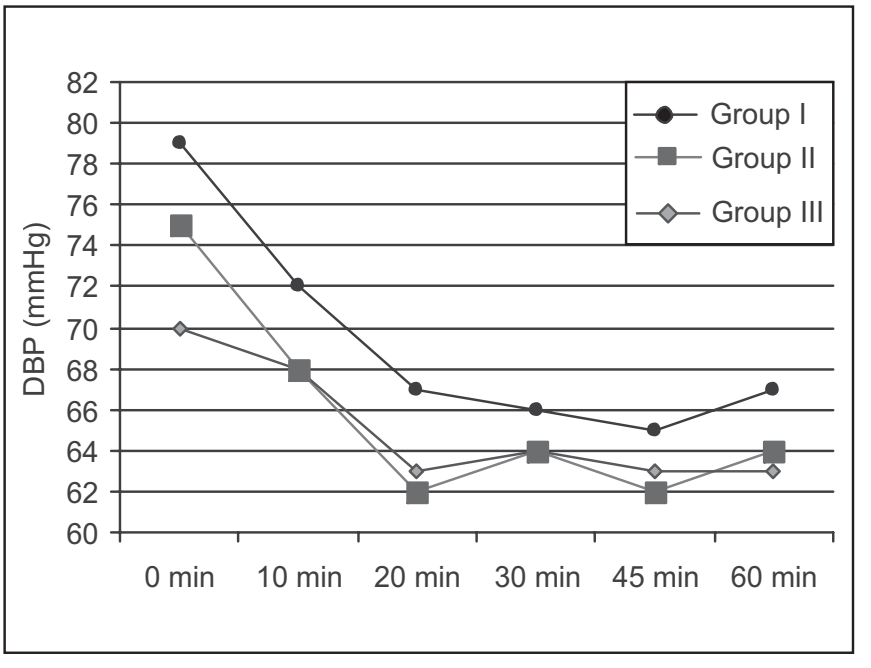

Figure 2 - Diastolic Blood Pressure Variation among Studied Groups

There was also a diastolic pressure decrease in the first 20 minutes, with no statistically significant difference among groups $(p=0.15)$ (Figure 2$)$ as compared to baseline values though. 
Heart rate analysis (Figure 3 ) showed that, although group II had a greater increase at 10 minutes, there were no statistically significant differences among groups throughout the post-anesthesia period ( $p=0.619$ ).

Muscle relaxation was evaluated by Bromage scale (Figure 4) at 30 minutes after local anesthetic injection. There were statistically significant differences in level 2 blockade in groups II and III as compared to group I.

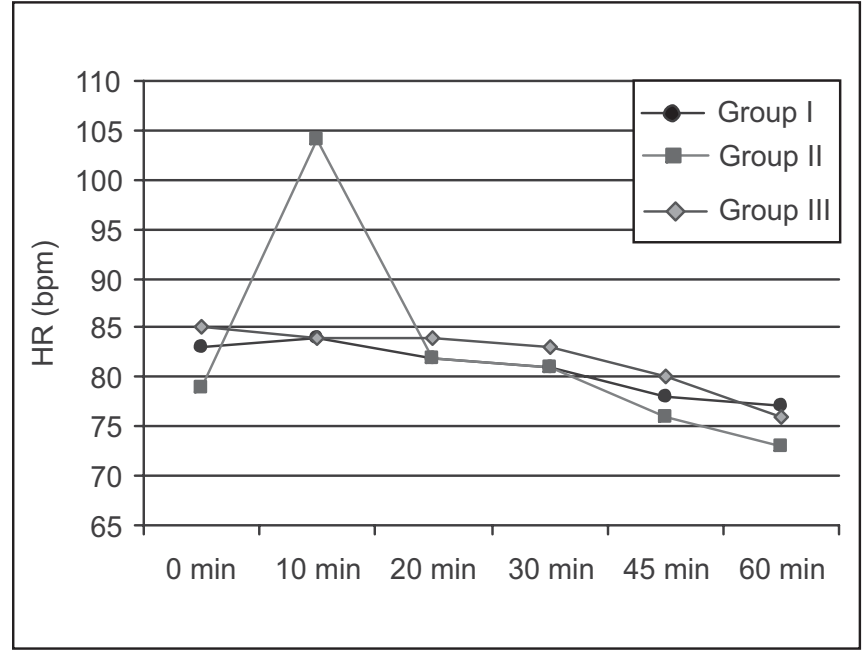

Figure 3 - Heart Rate Variation among Studied Groups

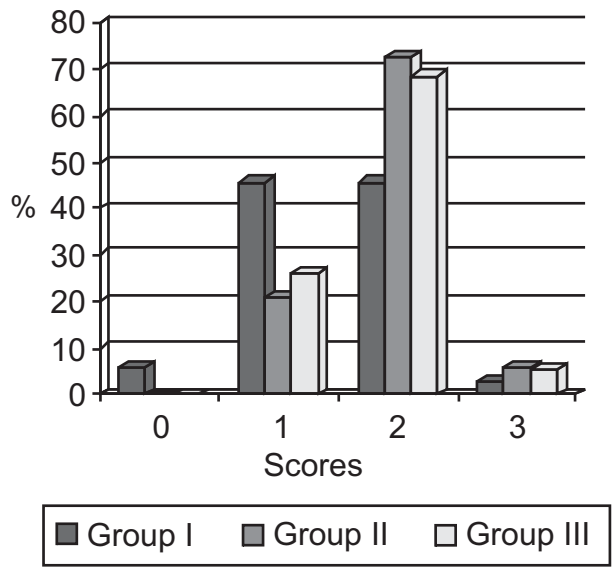

Figure 4 - Motor Block Levels (Bromage) at 30 Minutes

There was no statistically significant sensory block extension difference between groups along time (Figure 5).

Five group I patients, three group II patients and two group III patients received ephedrine due to blood pressure decrease to predefined levels for treatment. Anesthesia was insufficient for surgery performance in 3 patients in group I, 1 in group II and 2 in group III. Failures were partial, resolved in all patients with additional local anesthetics doses and sedation.

Revista Brasileira de Anestesiologia

Vol. 55, Nº 6, Novembro - Dezembro, 2005

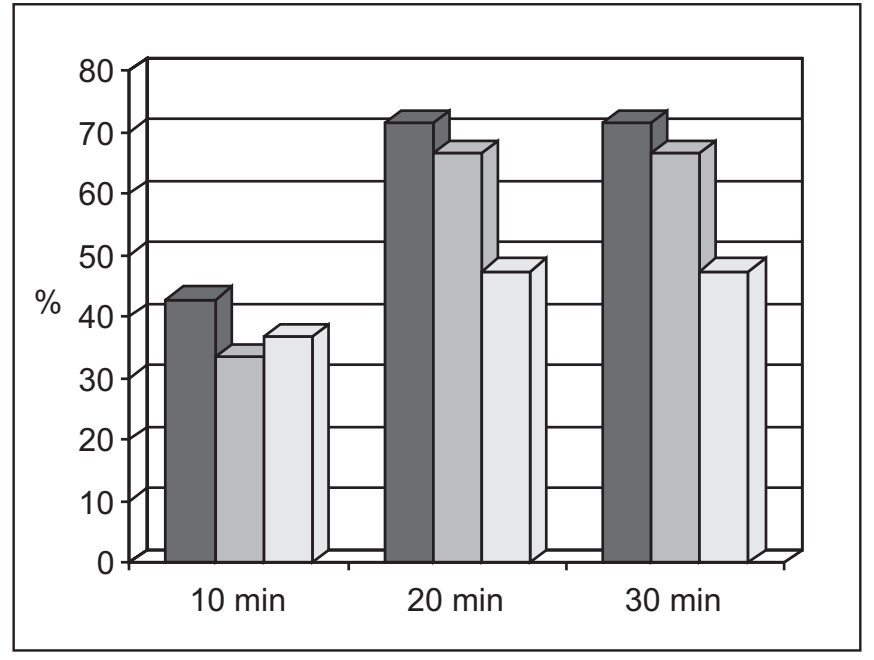

Figure 5 - Sensory Block above $T_{4}$

\section{DISCUSSION}

Our study has shown demographic homogeneity among groups, except for older age in group I. Although this is probably a casual finding due to sample size, age alone has minor influence in epidural block of adults, being important for pediatric and elderly populations ${ }^{5}$.

There were no statistically significant differences in hemodynamic parameters 10 minutes after local anesthetic injection. Difference in baseline systolic values between groups was probably due to the fact that patients were not premedicated. Heart rate, systolic and diastolic blood pressures had similar decreases, as it was to be expected, due to extensive sympathetic block, as shown by observed sensory block levels. Some patients in each group needed ephedrine to treat hypotension. Results are similar to other studies using $50 \%$ enantiomeric excess bupivacaine for lumbar ${ }^{6}$ or thoracic ${ }^{7}$ epidural anesthesia.

Epinephrine added to local anesthetic solution promotes local vasoconstriction, interfering with the absorption rate of the injected drug, thus allowing it to remain for a longer period in contact with nervous roots 5 . However, a study by Newton et al. has shown higher vasoconstrictor activity with the levogyrous isomer, factor considered responsible for long sensory block duration ${ }^{8}$. Our study has determined that all solutions should contain the vasoconstrictor agent because investigators were blind to the local anesthetic being used.

According to studies with the levogyrous isomer and $50 \%$ enantiomeric excess bupivacaine in patients submitted to cesarean sections $^{9-11}$, the association of opioid to local anesthetic potentiates its effects and provides adequate conditions for abdominal procedures. Another tactic described to prevent failures ${ }^{12}$ is increasing local anesthetic concentration to obtain the same effect, although this may expose patients to higher anesthetic mass, diminishing the advantages of low systemic toxicity drugs. In our study, fentanyl addition 
and even the placement of an epidural catheter for further local anesthetic injection have not prevented blockade failures, which may be related to the fact that anesthesia was performed by resident physicians.

Comparing isomers to racemic bupivacaine, an in vitro study

${ }^{13}$ has shown that the dextrogyrous one is more potent (1.36), the levogyrous is less potent $(0.45)$ and the $50 \%$ enantiomeric excess bupivacaine has similar potency (0.88). However, the clinical use of local anesthetics is more complex than what has been shown in animals, and in vivo potencies do not correlate to in vitro results ${ }^{14}$. Local factors affecting anesthetic spread and block extension are of major impact on clinical effects. The clinical use may not require absolute action potential suppression, but just the interruption of information coded by the depolarization pattern. Hence, the relative potency of epidural racemic bupivacaine and levobupivacaine would be the same ${ }^{15}$. These data validate the clinical correlation shown in our study in which local anesthetics are clinically comparable in the epidural space in equal concentrations and volumes.

This study has determined that surgery would only start 30 minutes after epidural injection to allow the evaluation of muscle relaxation. Mean time of 20 minutes was needed for motor block to reach the highest degree with racemic and $50 \%$ enantiomeric excess bupivacaine ${ }^{16}$. In a study by Cox 17 , results were similar between levobupivacaine and $0.5 \%$ racemic bupivacaine. Differences in motor block intensity in our groups were significant, with more patients presenting Bromage 2 in groups II and III as compared to group I. Delfino et al. ${ }^{18}$, studying pure $0.5 \%$ levogyrous isomer as compared to $50 \%$ enantiomeric excess mixture in patients submitted to varicose vein procedures, have also observed lower degree of relaxation, however difference was significant to Bromage levels 0 and 3 . This may be justified by the larger sample size of our study.

In conclusion, both levobupivacaine and $50 \%$ enantiomeric excess bupivacaine are local anesthetic solutions suitable for lower abdominal surgeries and clinically comparable to racemic bupivacaine. Levobupivacaine has promoted less motor block as compared to the other two solutions.

\section{REFERÊNCIAS - REFERENCES}

01. Simonetti MPB, Batista RA, Ferreira FMC - Estereoisomeria: a interface da tecnologia industrial de medicamentos e da racionalização terapêutica. Rev Bras Anestesiol, 1999;48: 390-399.

02. Mathias R, Torres MLA, Moraes JE et al - Comparação da qualidade da anestesia peridural para cesarianas com bupivacaína racêmica, levobupivacaína e ropivacaína. Rev Bras Anestesiol, 1997;47:(Supp23):CBA 012.

03. Simonetti MPB - Anestésicos Locais Espinhais Levógiros, em: Vale N, Delfino J - Anestesia Peridural: Atualização e Perspectiva. São Paulo, Editora Atheneu, 2000;81-92.
04. Bromage PR - A comparison of the hydrochloride and carbon dioxide salts of lidocaine and prilocaine in epidural analgesia. Acta Anaesthesiol Scand, 1965;16:(Suppl)55-69.

05. Gouveia MA, Labrunie G - Fatores que Influenciam o Bloqueio Peridural, em: Vale N, Delfino J - Anestesia Peridural: Atualização e Perspectiva. São Paulo, Editora Atheneu, 2000;120.

06. Gonçalves RF, Lauretti GR, Mattos AL - Estudo comparativo entre a bupivacaína racêmica a $0,5 \%$ e mistura enatiomérica de bupivacaína (S75-R25) em anestesia peridural. Rev Bras Anestesiol, 2003;53:169-176.

07. Borba DA, Araújo JH, Leão DG et al - Comparação clínica da mistura $75 \%$ do enantiomêro levógiro de $25 \%$ do enantiomêro dextrógiro da bupivacaína com a forma racêmica em anestesia peridural torácica. Rev Bras Anestesiol, 2001;51:(Supp27): CBA 017.

08. Newton DJ, Burke D, Khan F et al - Skin blood flow changes in response to intradermal injection of bupivacaine and levobupivacaine, assessed by laser Doppler imaging. Reg Anesth Pain Med, 2000;25:626-631.

09. Delfino J, Vale NB - Levobupivacaína em volumes fixos e concentrações diferentes associada a opióides em anestesia peridural para cesarianas. Rev Bras Anestesiol, 2000;50: 437-441.

10. Batista JBS, Oliveira JR - Estudo comparativo entre bupivacaína racêmica a $0,5 \%$ e a mistura enantiomérica de bupivacaína (S75-R25) a 0,5\% associadas ao sufentanil na anestesia peridural para cesariana. Rev Bras Anestesiol, 2002; 52:(Suppl):CBA 060B.

11. Cortes CAF, Oliveira AS, Castro LFL et al - Estudo comparativo entre bupivacaína a $0,5 \%$, mistura enantiomérica de bupivacaína (S75-R25) a $0,5 \%$ e ropivacaína a $0,75 \%$ associadas ao fentanil em anestesia peridural para cesarianas. Rev Bras Anestesiol, 2003;53:177-187.

12. Kopacz DJ, Allen HW, Thompson GE - A comparison of epidural levobupivacaine $0.75 \%$ with racemic bupivacaine for lower abdominal surgery. Anesth Analg, 2000;90:642-648.

13. Sudo R, Trachez M, Sudo $G$ et al - Comparative motor nerve block between bupivacaine enantiomers and $\mathrm{S}(-)$ ropivacaine. Anesthesiol, 2001;97:A74.

14. Pateromichelakis S, Prokopiou AA - Local anesthesia efficacy: discrepancy between in vitro and in vivo studies. Acta Anaesthesiol Scand, 1988;32:672-675.

15. Salinas FV, Liu SL, Scholz AM - Analgesics, em: Evers AS, Maze $M$ - Anesthetic Pharmacology. Philadelphia, Churchill Livingstone, 2004;517-519.

16. Tanaka PP, Salvalaggio MF, Souza R et al - Estudo comparativo entre a bupivacaína a $0,5 \%$ e a mistura enantiomérica de bupivacaína (S75-R25) a 0,5\% em anestesia peridural em pacientes submetidos à cirurgia ortopédica de membros inferiores. Rev Bras Anestesiol, 2003;53:331-337.

17. Cox CR, Faccenda KA, Gilhooly C et al - Extradural S (-) bupivacaine: comparison with racemic RS-bupivacaine. $\mathrm{Br} \mathrm{J}$ Anaesth, 1998;80:289-293.

18. Delfino J, Vale NB - Bupivacaína levógira a $0,5 \%$ pura versus mistura enantiomérica de bupivacaína (S75-R25) a 0,5\% em anestesia peridural para cirurgia de varizes. Rev Bras Anestesiol, 2001;51:474-482. 


\section{RESUMEN}

Tanaka PP, Ogleari M, Valmorbida P, Tanaka MAA - Estudio Comparativo entre Soluciones a $0,5 \%$ de Levobupivacaína, Bupivacaína en Exceso Enantiomérico del 50\% y Bupivacaína Racémica en Anestesia Peridural para Cirugía de Abdomen Inferior

JUSTIFICATIVA Y OBJETIVOS: Con la finalidad de encontrar un anestésico local más seguro que la bupivacaína, varios estudios en animales fueron realizados con sus isómeros. Este estudio tuvo como objetivo evaluar la eficacia de la bupivacaína en exceso enantiomérico del $50 \%$, comparada a la levobupivacaína y a la bupivacaína racémica, en la anestesia peridural en pacientes sometidos a la cirugía de abdomen inferior, por el período de una hora después de la inyección de las soluciones.

MÉTODO: Después de la aprobación por el Comité de Ética en Pesquisa, participaron de este estudio, eventual y doblemente encubierto, 87 pacientes con edad entre 18 y 65 años, estado físico ASA I y II sometidos a cirugía del abdomen inferior. Fueron distribuidos en tres grupos que fraccionadamente recibieron solución conteniendo $27 \mathrm{~mL}$ (incluyendo la dosis-test) de anestésico local con adrenalina (1:200.000) y fentanil (100 $\mu \mathrm{g})$. El grupo I recibió solución de levobupivacaína a 0,5\%, el grupo II recibió solución de bupivacaína en exceso enantiomérico del $50 \%$ a 0,5\% y el grupo III recibió solución de bupivacaína a $0,5 \%$. Los pacientes fueron monitorizados por medio de oxímetro de pulso, cardioscopio y presión arterial no invasiva. Fueron investigadas las características motoras y sensitivas del bloqueo anestésico, bien como la incidencia de efectos colaterales. Los frascos de anestésico local fueron preparados sin identificación, numerados y solamente al final del estudio la lista de distribución eventual fue abierta.

RESULTADOS: No se observaron diferencias significativas con relación a la altura y estado físico. Diferencia demográfica significativa fue encontrada con relación a la edad en el grupo I. Los parámetros hemodinámicos fueron semejantes entre los grupos. Hubo una diferencia significativa con relación a la intensidad del bloqueo motor relatado entre los grupos estudiados (menor intensidad en el grupo I comparada a los grupos II y III).

CONCLUSIONES: Fue observado un adecuado bloqueo motor y sensitivo para la realización de la cirugía en los grupos estudiados con pocos efectos colaterales, sugiriendo que las soluciones son eficaces en la anestesia peridural para cirugía de abdomen inferior. La levobupivacaína presentó menor bloqueo motor que los otros dos grupos. 